\title{
A New Canonical Form for Fast Boolean Matching in Logic Synthesis and Verification
}

\author{
Afshin Abdollahi \\ University of Southern California \\ afshin@usc.edu
}

\begin{abstract}
An efficient and compact canonical form is proposed for the Boolean matching problem under permutation and complementation of variables. In addition an efficient algorithm for computing the proposed canonical form is provided. The efficiency of the algorithm allows it to be applicable to large complex Boolean functions with no limitation on the number of input variables as apposed to previous approaches, which are not capable of handling functions with more than seven inputs. Generalized signatures are used to define and compute the canonical form while symmetry of variables is used to minimize the computational complexity of the algorithm. Experimental results demonstrate the efficiency and applicability of the proposed canonical form.
\end{abstract}

\section{Categories \& Subject Descriptors}

B.6.3 [Hardware] Logic Design: Design aids - Automatic synthesis, Optimization, verification.

\section{General Terms}

Algorithms, Design, Verification.

\section{Introduction}

Boolean matching is the problem of determining whether a Boolean function can be functionally equivalent to another one under a permutation of its inputs and complementation of some of its inputs. Boolean matching algorithms have many applications in logic synthesis including cell-library binding where it is necessary to repeatedly determine whether some part (cluster) of a Boolean network can be realized by any of the cells in a library [1]. Boolean matching is a critical and CPU-intensive task and therefore, there have been many efforts to effectively solve the problem [2]. Boolean functions that are equivalent under negation of inputs are $\mathrm{N}$-equivalent, under permutation of inputs are $\mathrm{P}$ equivalent, and under both stated conditions, are NP-equivalent [3]. An exhaustive method for Boolean matching is computationally expensive since the complexity of such an algorithm for $n$-variable functions is $O\left(n ! 2^{n}\right)$.

Boolean matching algorithms can be classified into two categories: pair-wise matching algorithms and algorithms based on canonical forms of functions. Pair-wise Boolean matching algorithms are based on a semi-exhaustive search where the search space is pruned by the use of some signatures which are computed from some properties of Boolean functions [2]. A signature in general is a description of (one or more) input variables of a Boolean function that is independent of the permutation or complementation of the variables of the function. To match a function against a cell library, pair-wise matching algorithms often need to perform pair-wise matching of the function with all the library cells. Therefore, these algorithms can only handle libraries with a modest size.

Permission to make digital or hard copies of all or part of this work for personal or classroom use is granted without fee provided that copies are not made or distributed for profit or commercial advantage and that copies bear this notice and the full citation on the first page. To copy otherwise, or republish, to post on servers or to redistribute to lists, requires prior specific permission and/or a fee.

DAC 2005, June 13-17, 2005, Anaheim, California, USA.

Copyright 2005 ACM 1-59593-058-2/05/0006...\$5.00.

\author{
Massoud Pedram \\ University of Southern California \\ pedram@ceng.usc.edu
}

Boolean matching algorithms that belong to the second category compute some canonical form for Boolean functions [5] - [10]. These algorithms are based on the fact that two functions match if and only if their canonical forms are the same. Burch and Long introduced a canonical form for matching under complementation and a semi-canonical form for matching under permutation of the variables [5]. Thus, in order to handle complementation and permutation of inputs simultaneously, a large number of forms for each cell are required. Other researchers, including Wu et al. [6], Debnath and Sasao [8], and Ciric and Sechen [9] also proposed canonical forms that are applicable for Boolean matching under permutation of the variables only and do not handle complementation of inputs. Hinsberger and Kolla [7] and Debnath and Sasao [10], introduced a canonical form for solving the general Boolean matching problem. However their approach is mainly based on manipulating the truth table of the function and using a table look-up which introduces an enormous space complexity, thus limiting the algorithm to library cells with seven and fewer input variables, which is a major limitation.

In this paper a new canonical form for representing Boolean functions is introduced. The proposed canonical form for an arbitrary Boolean function is the unique Boolean function that is obtained after applying some CP transformation on the input variables. The canonical forms of NP-equivalent Boolean functions are identical. Next, an effective technique is presented for generating this canonical form. The proposed method is based on using generalized signatures (signatures of one or more variables) to find a canonicity-producing $(\mathrm{CP})$ phase assignment and ordering for variables. From here on, phase assignment and ordering for variables is referred to as a transformation on variables. For most Boolean functions, single-variable and twovariable signatures are enough to recognize all variables (i.e., to obtain a CP transformation.) However, use of single-variable and two-variable signatures alone may not result on a canonical input transformation. In this paper it is shown that, by using generalized signatures of one or more variables, it is always possible to create a CP transformation on variables of the function.

Experimental results provided in this paper demonstrate that the proposed approach for computing the canonical form does not have the limitations of previous works; i.e. it computes the canonical form under both permutation and complementation of variables and there is no limit on number of variables. An important advantage of the proposed technique is the way it handles and uses the symmetry of variables to minimize the complexity of the algorithm compared to some of the previous approaches which are not able to consider symmetries [7][10]. Hence, the proposed technique is applicable to large libraries with cells of any number of inputs. Also it can be used for logic verification of large circuits since there is no limit on the number of input variables. In section II, definitions and terminology are introduced. In section III, CP transformations and canonical forms are defined. Section IV describes how symmetries of variables are handled and section $\mathrm{V}$ provides the details of computing the canonical form followed by experimental results and conclusions in sections VI and VII.

\section{Preliminaries}

Let $X$ denote a vector $\left(x_{1}, x_{2}, \ldots, x_{\mathrm{n}}\right)$ of Boolean variables and $f(X)$ a single-output completely-specified Boolean function of $X$. A 
literal is a variable, $x_{i}$, or its complement $\bar{x}_{i}$. In general a literal can be denoted as $x_{i}^{p}$, where $p$ denotes the phase assignment to variable $x_{i}$ i.e., $x_{i}^{1}=x_{i}$ and $x_{i}^{0}=\bar{x}_{i}$. A minterm is a vertex in the $n$ dimensional Boolean space, $\{0,1\}^{n}$ and a cube is the conjunction of some literals. An NP transformation, $\Gamma$, is an onto mapping which assigns a unique phase and variable ordering to each variable in $X$ i.e., $\Gamma(X)=T$ where $T=\left(t_{1}, t_{2}, \ldots, t_{n}\right)$ and $t_{i}=x_{\pi(i)}^{p(i)}$. Here, $\pi(i)$ denotes the position of $x_{i}$ under permutation $\pi$. Similarly, $p(i)$ denotes the phase of $x_{\pi(i)}$ under phase assignment $p$. The inverse NP transformation $\Gamma^{-1}$ applied to vector $T$ produces vector $X$. For example, assume that a transformation $\Gamma$ on $\left(x_{1}, x_{2}, x_{3}\right)$ produces $\left(x_{2}, \bar{x}_{3}, x_{1}\right)$. The inverse transformation $\Gamma^{-1}$ on $\left(x_{2}, \bar{x}_{3}, x_{1}\right)$ produces $\left(x_{1}, x_{2}, x_{3}\right)$. Notice also that $\Gamma^{-1}\left(x_{1}, x_{2}, x_{3}\right)=\left(x_{3}, x_{1}, \bar{x}_{2}\right)$.

Fact. Boolean functions $f_{1}(X)$ and $f_{2}(X)$ are $N P$-equivalent exactly if there exists a transformation $\Gamma(X)=T$ such that $f_{1}(X)=f_{2}(T)$. Notice that if $f_{1}(X)=f_{2}(T)$ then $f_{1}\left(T^{-1}\right)=f_{2}(X)$ where $T^{-1}=\Gamma^{-1}(X)$.

Note that NP-equivalence is an equivalence relation, partitioning the space of n-input Boolean functions to equivalence classes.

Example 1. Let $f_{1}\left(x_{1}, x_{2}, x_{3}\right)=x_{1} x_{2}+\bar{x}_{1} x_{3}$ and $f_{2}\left(x_{1}, x_{2}, x_{3}\right)=$ $x_{1} x_{3}+\bar{x}_{2} \bar{x}_{3}$. Then $f_{1}(X)=f_{2}(T)$ where $X=\left(x_{1}, x_{2}, x_{3}\right)$ and $T=\left(x_{2}, \bar{x}_{3}, x_{1}\right)$. Thus, $f_{1}(X)$ and $f_{2}(X)$ are NP-equivalent.

In the remainder of this paper, for a given $X$, when there is no confusion, we will use $T$ to denote the transformation, $\Gamma$, or its result, $\Gamma(X)$. A similar overloading will be used for $T^{-1}$.

For any function $f(X)$, let $|f(X)|$ denote the number of minterms covered by $f(X)$. The cofactor of $f$ with respect to a literal $x_{i}\left(\bar{x}_{i}\right)$ is the Boolean function obtained by setting $x_{i}\left(\bar{x}_{i}\right)$ to 1 in $f$ and is denoted by $f_{x_{i}}\left(f_{\bar{x}_{i}}\right)$, which is considered as a function with the same number of input variables as $f$. The cofactor of $f$ with respect to a cube, $b$, is the Boolean function obtained by setting all literals of $b$ to 1 in $f$ and is denoted by $f_{b}$, which is also considered as a function with the same number of input variables as $f$.

Definition: For any cube $b$, generalized signature, $\left|f_{b}\right|$, of function $f$ with respect to cube $b$ is equal to the number of minterms in the onset of function $f_{b}$. If $b$ consists of exactly $k$ literals, then the corresponding signature will be referred to as a $k^{\text {th }}$ order signature.

\section{Canonical Form}

There are $2^{n} n$ ! NP transformations on $n$ variables $X=\left(x_{1}, x_{2}, \ldots, x_{n}\right)$ of function $f(X)$. Some of these transformations result in identical Boolean functions. Therefore, the set of Boolean functions of exactly $n$ variables can be partitioned into a set of NP-equivalent functions. Each equivalence class is uniquely represented by a single NP-representative function. It is important to be able to determine the equivalence class of a given Boolean function, $f(X)$. This is in turn achieved by applying a transformation to $X$ such that $f(T)$ is the NP-representative function of that class.

Definition: The canonical form of function $f(X)$ is a function, $f^{C}$, such that $f^{C}(X)=f\left(T_{C}\right)$, where $T_{C}$ denotes a canonicityproducing (or CP) transformation. Notice that $f^{C}\left(T_{C}^{-1}\right)=f(X)$.

There may be more than one such $\mathrm{CP}$ transformation due to variable symmetries as described in the next section. For example, for function $f(X), \quad T_{C_{1}}$ and $T_{C_{2}}$ may be two different $\mathrm{CP}$ transformations, which result in the same canonical form $f^{C}(X)$, i.e., $f\left(T_{C_{1}}\right)=f\left(T_{C_{2}}\right)=f^{C}(X)$. To guarantee that the canonical forms of $f(X)$ and its negation-equivalent functions are the same, constraint $\left|f_{\bar{t}_{i}}\right| \leq\left|f_{t_{i}}\right|$ is imposed on the inverse of $\mathrm{CP}$ transformation, $T$. In addition, for computational efficiency reasons, "NE-symmetric" variables (cf. section IV) must be placed next to one another. Therefore, an order relation, ' $\prec$ ', is defined among the transformations that satisfy these constraints.

Definition: For a transformation $T=\left(t_{1}, t_{2}, \ldots, t_{n}\right)$, the signature vector $S^{T}$ is defined as:

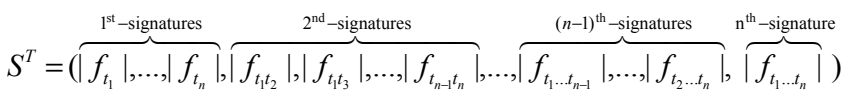
Signature vector $S^{T}$ includes the $1^{\text {st }}$-signatures followed by the $2^{\text {nd }}$ and higher order signatures up to the $n^{\text {th }}$-signatures. Signatures of $S^{T}$ correspond to cofactors of $f$ with respect to cubes consisting of non-empty subsets of vector $T$. (Only positive phases of $t_{i}$ are considered; hence $S^{T}$ includes $2^{n}-1$ signatures.)

Definition: The relation ' $\prec$ ' between $T_{1}$ and $T_{2}$ is defined based on the lexicographic comparison of their corresponding signature vectors, $S^{T_{1}}$ and $S^{T_{2}}$, i.e., $T_{1} \prec T_{2} \Leftrightarrow S^{T_{1}} \prec S^{T_{2}}$.

Recall that in lexicographic comparison of two vectors, the corresponding entries are compared until an inequality is encountered. For example $(2,1,3,4) \prec(2,3,0,1)$. Notice that because $|f|$ is invariant under a CP transformation on its variables, it is not included in the signature vector.

Definition: The maximal transformation is a transformation which is maximal with respect to the order relation, ' $\prec$ ' in the set of all transformations that satisfy the aforesaid constraints.

A necessary condition for this method to be valid is that when two signature vectors $S^{T_{1}}$ and $S^{T_{2}}$ are identical, the corresponding Boolean functions $f\left(T_{1}^{-1}\right)$ and $f\left(T_{2}^{-1}\right)$ will also be identical, i.e., $S^{T_{1}}=S^{T_{2}} \Leftrightarrow f\left(T_{1}^{-1}\right)=f\left(T_{2}^{-1}\right)$ as stated equivalently in theorem 1 .

Theorem 1. For a transformation $T^{-1}$ on variables $X$ of a function $f$, values of $|f|$ and signature vector $S^{T}$ uniquely and completely specify function $f$.

Proof: Let vector $F$ represent the values of function $f$ in all $2^{n}$ minterms (the last column of the truth table of f.) Then the vector $\mathrm{S}=\left[|f|, S^{T}\right]$ can be obtained using the matrix relation $\mathrm{S}=\mathrm{A} \times \mathrm{F}$ where $\mathrm{A}$ is a reversible matrix of 0 and 1 entries and values and operations in this matrix relation are regarded as integers (rather than Boolean.) The proof follows from the reversibility of matrix A. Details are however omitted because of space limitation.

When comparing two different transformations $T_{1}$ and $T_{2}$, it is often unnecessary to completely compute all the signature vectors. More precisely, if $f\left(T_{1}^{-1}\right) \neq f\left(T_{2}^{-1}\right)$, then most of the time the $1^{\text {st }}$ signatures alone will determine the order between $T_{1}$ and $T_{2}$. However, if all of the $1^{\text {st }}$ signatures are equal, then the $2^{\text {nd }}$ signatures should be compared. Subsequently, if the $2^{\text {nd }}$-signatures are also equal, then the $3^{\text {rd }}$-signatures must be compared, and so on until an inequality is encountered. Based on theorem 1 it is guaranteed that if $f\left(T_{1}^{-1}\right) \neq f\left(T_{2}^{-1}\right)$, then $S^{T_{1}} \neq S^{T_{2}}$ i.e., an inequality in some of signatures will always occur. Experimental evidence shows that in the great majority of cases, a signature inequality occurs for the low order signatures $\left(1^{\text {st }}\right.$ and $2^{\text {nd }}$ signatures.) Intuitively, the reason is that the lower order signatures depend on more minterms of the function and thus contain more information about the function. For example a $1^{\text {st }}$-signatre depends on $2^{\text {n-1 }}$ minterms which is a half of the whole Boolean space $\left(2^{\mathrm{n}}\right.$ minterms) whereas a $2^{\text {nd }}$-signatre depends on one-forth of all minterms $\left(2^{\mathrm{n}-2}\right.$ minterms.) Hence, the $1^{\mathrm{st}}$-signatures are the most powerful and effective signatures. The $2^{\text {nd }}$-signatures are the next most effective signatures and so on. The reader will observe that this arrangement of the proposed signature vector minimizes the computational complexity.

In this paper we analyze and use the variable symmetries to significantly prune the search space, and thereby, avoid unnecessary computations. We to use the $1^{\text {st }}$ and $2^{\text {nd }}$ signatures to order (sort) the literals (variables after phase assignment) of the 
function. Subsequently, if the resultant ordering does not conclude a CP transformation, then the $3^{\text {rd }}$ and possibly higher order signatures are used to identify a CP transformation.

\section{Symmetry Classes}

Accounting for symmetry relations between variables is critical in minimizing the complexity of any Boolean matching algorithm. Hence, this paper focuses on efficient handling and utilization of symmetry relations and symmetry classes.

Definition: Given function $f(X)$, variables $x_{i}$ and $x_{j}$ are said to be non-equivalent-symmetric (NE-symmetric), denoted as $x_{i} \mathrm{NE} x_{j}$, if $f$ is invariant under swapping $x_{i}$ and $x_{j}$ [11] i.e., $f$ $\left(x_{1}, \ldots, x_{i}, \ldots, x_{j}, \ldots, x_{n}\right)=f\left(x_{1}, \ldots, x_{j}, \ldots, x_{i}, \ldots, x_{n}\right)$. Notice that $x_{i} \mathrm{NE} x_{j}$ if and only if $f_{x_{i} \bar{x}_{j}}=f_{\bar{x}_{i} x_{j}}$. Two variables $x_{i}$ and $x_{j}$ of $f(X)$ are said to be equivalent-symmetric (E-symmetric), denoted as $x_{i} \mathrm{E} x_{j}$, if $x_{i} \mathrm{NE} \bar{x}_{j}$. Notice that $x_{i} \mathrm{E} x_{j}$ if and only if $f_{\bar{x}_{i} \bar{x}_{j}}=f_{x_{i} x_{j}}$.

Two variables $x_{i}$ and $x_{j}$ of $f(X)$ are called symmetric, denoted as $x_{i} \mathrm{~S} x_{j}$, if $x_{i} \mathrm{NE} x_{j}$ or $x_{i} \mathrm{E} x_{j}$.

Fact: Transitive property. If $x_{i} \mathrm{~S} x_{j}$ and $x_{j} \mathrm{~S} x_{k}$, then $x_{i} \mathrm{~S} x_{k}$.

Since symmetric relation, $\mathrm{S}$, is an equivalence relation, the set of variables, $x_{1}, x_{2}, \ldots, x_{n}$ can be divided into equivalent classes $C_{1}, C_{2}, \ldots, C_{m}$, that are referred to as symmetry classes. Similarly it is possible to define NE-symmetry relation and NE-symmetry classes (NE-classes) with respect to non-equivalent symmetries.

Definition: A symmetry (or NE-symmetry) class is maximal if no other variable is symmetric (or NE-symmetric) to the variables of the class.

Example 2. Consider the Boolean function: $f\left(x_{1}, x_{2}, x_{3}, x_{4}, x_{5}\right)=$ $\left(x_{1}+\bar{x}_{3}+x_{4}\right)\left(\bar{x}_{2}+x_{5}\right)$. Variables $x_{1}, x_{2}, x_{3}, x_{4}, x_{5}$ can be divided into two symmetry classes: $C_{1}=\left\{x_{1}, x_{3}, x_{4}\right\}$ and $C_{2}=\left\{x_{2}, x_{5}\right\}$. Note that a symmetry class $\left(C_{1}\right)$ may include both $\mathrm{NE}$ and $\mathrm{E}$ symmetries (i.e., $x_{1} \mathrm{E} x_{3}$ and $x_{1} \mathrm{NE} x_{4}$.)

Lemma 1. Given a symmetry class $C=\left\{x_{1} \ldots, x_{i}\right\}$ of function $f(X)$, there always exists a phase assignment $P=\left(p_{1}, \ldots, p_{i}\right)$ to variables $\left(x_{1}, \ldots, x_{i}\right)$ such that $C^{P}=\left\{x_{1}^{p_{1}}, \ldots, x_{i}^{p_{i}}\right\}$ is an NE-class.

Proof: The proof is by construction. The construction algorithm (which we refer to as the Generate-NE-symmetry) starts by setting $C^{P}=\left\{x_{1}\right\}$, i.e., $p_{1}=1$. In the next step, if $x_{1} \mathrm{NE} x_{2}$, then $x_{2}$ is added to $C^{P}$, resulting in $C^{P}=\left\{x_{1}, x_{2}\right\}$, i.e., $p_{2}=1$. However, if $x_{1} \mathrm{E} x_{2}$, then $C^{P}=\left\{x_{1}, \bar{x}_{2}\right\}$, i.e., $p_{2}=0$. At each step of the algorithm, $C^{P}$ remains NE-symmetric by assigning appropriate phases to variables being added to $C^{P}$. Consider step $j$ where $j-1$ literals have been included in the NE-class i.e., $C^{P}=\left\{x_{1}{ }^{p_{1}}, x_{2}{ }^{p_{2}}, \ldots, x_{j-1}{ }^{p_{j-1}}\right\}$. Now $x_{j}$ is either NE-symmetric with all members of $C^{P}=\left\{x_{1}{ }^{p_{1}}\right.$, $\left.x_{2}{ }^{p_{2}}, \ldots, x_{j-1}^{p_{j-1}}\right\}$ or E-symmetric with all of them. In the first case $x_{j}$ is added to $C^{P}\left(p_{j}=1\right)$ while in the second case $\bar{x}_{j}$ is added to $C^{P}\left(p_{j}=0\right)$ resulting in $C^{P}=\left\{x_{1}^{p_{1}}, \ldots, x_{j-1}^{p_{j-1}}, x_{j}^{p_{j}}\right\}$, which is an NE-class. The algorithm continues until all variables of $C$ are added to $C^{P}$ with appropriate phases.

The cofactor of function $f$ with respect to any literal in an NE-class of $f, C=\left\{x_{1}, x_{2}, \ldots, x_{i}\right\}$, is a unique function i.e., $f_{x_{1}}=f_{x_{2}}=\ldots=f_{x_{i}}$. Therefore, the cofactor of $f$ with respect to the NE-class $C$ can be defined as the cofactor of $f$ with respect to any literal from $C$, e.g., $f_{C}=f_{x_{1}}$. The second order cofactor of $f$ with respect to NEclasses $C_{1}$ and $C_{2}$ (i.e., $f_{C_{1} C_{2}}$ ) is defined similarly.

Definition: The $1^{\text {st }}$-order signature $\left(1^{\text {st }}\right.$-signature) of $f$ with respect to an NE-class $C$ is defined as $\left|f_{C}\right|$ whereas the second order signature ( $2^{\text {nd }}$-signature) of $f$ with respect to NE-classes $C_{1}$ and $C_{2}$ is defined as $\left|f_{C_{1} C_{2}}\right|$. Higher order signatures are similarly defined.

\section{Computing the Canonical Form}

In this section, an algorithm, called compute-CF, for computing the canonical form of function $f(X)$ is described. The algorithm consists of several steps including classifying variables to symmetry classes, phase assignment, using $1^{\text {st }}$ and $2^{\text {nd }}$ signatures (and if necessary higher order signatures) to order classes.

As stated previously, compute-CF finds a CP transformation which is the inverse of the maximal transformation among the $2^{n} n$ ! possible transformations. Note that there may exist more than one maximal transformation for any function $f$; however, all such maximal transformations result in the same unique canonical form. Recall that function $f$ will remain invariant under permutations inside an NE-symmetry class. Therefore, instead of finding a transformation on the variables, variables are first partitioned into maximal symmetry classes, next maximal NE-classes $C_{1}, C_{2}, \ldots, C_{m}$ are generated by appropriate phase assignment to members of each class using the Generate-NE-symmetry provided in the proof of lemma 1. Next the $1^{\text {st }}$-signatures are used to construct a maximal transformation on NE-classes if possible. If the $1^{\text {st }}$-signatures do not contain enough information to generate a maximal transformation, then the $2^{\text {nd }}$-sinatures (and possibly higher order signatures) will be used to find a maximal transformation.

Example 3. Consider function $f$ in example 2. After applying the Generate-NE-symmetry, the NE-classes are: $C_{1}=\left\{x_{1}, \bar{x}_{3}, x_{4}\right\}$ and $C_{2}=\left\{x_{2}, \bar{x}_{5}\right\}$. Because $f$ is a function of five variables, there exists $2^{5} 5 !=3840$ transformations on variables. However, since $f$ is invariant with respect to permutations inside $C_{1}$ or $C_{2}$, there are only $2^{2} 2 !=8$ distinct transformations, which result from transformations on NE-classes $C_{1}$ and $C_{2}$ i.e., $\left(C_{1}, C_{2}\right),\left(C_{1}, \bar{C}_{2}\right)$, $\left(\bar{C}_{1}, C_{2}\right),\left(\bar{C}_{1}, \bar{C}_{2}\right),\left(C_{2}, C_{1}\right),\left(C_{2}, \bar{C}_{1}\right),\left(\bar{C}_{2}, C_{1}\right)$, and $\left(\bar{C}_{2}, \bar{C}_{1}\right)$.

To be concise, from this point on, we will use the term "class" to refer to "NE-class" unless stated otherwise. Each transformation on classes determines a transformation on variables (among which the maximal transformation must be identified.) For example $\left(C_{2}, \bar{C}_{1}\right)$ corresponds to the transformation $\left(x_{2}, \bar{x}_{5}, \bar{x}_{1}, x_{3}, \bar{x}_{4}\right)$.

By classifying variables to classes and only considering transformations on the resulting classes, variable transformations (and thus, the maximal transformation) are restricted to transformations in which members of a class are NE-symmetric and are placed next to each other.

The next step of compute-CF is to assign a phase to each class $C_{i}$ by using the $1^{\text {st }}$-signatures of $f$ with respect to $C_{i}$ and the complement of $C_{i}$ as follows. (The complement of a class $C_{i}$, as the defined before, is a class $\bar{C}_{i}$ consisting of complements of literals from $C_{i}$.) If $\left|f_{\bar{C}_{i}}\right|<\left|f_{C_{i}}\right|$, then the positive phase $\left(C_{i}\right)$ is assigned to $C_{i}$; otherwise if $\left|f_{\bar{C}_{i}}\right|>\left|f_{C_{i}}\right|$, then the negative phase is assigned i.e., literals of $C_{i}$ will be negated. In case of equality, the phase of the class is marked undecided. The phase of undecided classes must be determined in subsequent steps of the algorithm.

Example 4. Continuing with examples 2 and 3 , the $1^{\text {st }}$-signatures of $f$ with respect to the classes and their complements are as follows: $\left|f_{C_{i}}\right|=12,\left|f_{\bar{C}_{i}}\right|=9,\left|f_{C_{2}}\right|=7,\left|f_{\bar{C}_{2}}\right|=14$. Since $\left|f_{\bar{C}_{1}}\right|<\left|f_{C_{1}}\right|$, positive phase is assigned for $C_{1}$ (i.e., $C_{1}=\left\{x_{1}, \bar{x}_{3}, x_{4}\right\}$.) Since $\left|f_{\bar{C}_{2}}\right|>\left|f_{C_{2}}\right|$, negative phase is assigned to $C_{2}$ (new $C_{2}=\left\{\bar{x}_{2}, x_{5}\right\}$.)

Let $C_{1}, C_{2}, \ldots, C_{m}$ represent the resulting classes after proper phase assignment. (In case of example 4, $C_{1}=\left\{x_{1}, \bar{x}_{3}, x_{4}\right\}$ and $C_{2}=\left\{\bar{x}_{2}, x_{5}\right\}$.) After phases are assigned, new classes are ordered based on their $1^{\text {st }}$-signatures. (The $1^{\text {st }}$ - signatures used for ordering are values after phase assignment.) Without loss of generality, 
let's assume that $\left|f_{C_{1}}\right| \leq\left|f_{C_{2}}\right| \leq \ldots \leq\left|f_{C_{m}}\right|$. Thus classes are ordered as $\left(C_{1}, C_{2}, \ldots, C_{m}\right)$. If the phase of a class $\left(C_{i}\right)$ is undecided, since $\left|f_{\bar{C}_{i}}\right|=\left|f_{C_{i}}\right|$, the phase of $C_{i}$ (positive or negative) will not affect the outcome of ordering (i.e., $\left|f_{C_{i}}\right|$ or $\left|f_{\bar{C}_{i}}\right|$ could be used for ordering). If the $1^{\text {st }}$-signatures are distinct values for $C_{1}, C_{2}, \ldots, C_{m}$, then a unique ordering can be achieved since $\left|f_{C_{1}}\right|<\left|f_{C_{2}}\right|<\ldots<\left|f_{C_{m}}\right|$.

Example 5. Continuing with example 4, $f\left(x_{1}, x_{2}, x_{3}, x_{4}, x_{5}\right)=$ $\left(x_{1}+\bar{x}_{3}+x_{4}\right)\left(\bar{x}_{2}+x_{5}\right)$; the classes after phase assignment will be $C_{1}=\left\{x_{1}, \bar{x}_{3}, x_{4}\right\} \quad$ and $C_{2}=\left\{\bar{x}_{2}, x_{5}\right\}$. Furthermore, $\left|f_{C_{1}}\right|=12<$ $\left|f_{C_{2}}\right|=14$. Thus, $\left(C_{1}, C_{2}\right) \equiv\left(x_{1}, \bar{x}_{3}, x_{4}, \bar{x}_{2}, x_{5}\right)$ will be a maximal transformation. The inverse of this maximal transformation is a CP transformation $T_{C}=\left(x_{1}, \bar{x}_{4}, \bar{x}_{2}, x_{3}, x_{5}\right)$ and the canonical form (cf. section III) is: $f^{C}(X)=f\left(T_{C}\right)=\left(x_{1}+x_{2}+x_{3}\right) \cdot\left(x_{4}+x_{5}\right)$.

It is important to notice that the application of the steps of the compute-CF algorithm described so far and also the next steps will produce the same canonical form for NE-equivalent functions. Example 6. Consider two Boolean functions: $f_{1}\left(x_{1}, x_{2}, x_{3}, x_{4}, x_{5}\right)$ $=\left(x_{1}+\bar{x}_{3}+x_{4}\right) \cdot\left(\bar{x}_{2}+x_{5}\right)$ and $f_{2}\left(x_{1}, x_{2}, x_{3}, x_{4}, x_{5}\right)=\left(x_{1}+x_{2}\right) \cdot\left(x_{3}+x_{4}+x_{5}\right)$. For function $f_{1}$ a CP transformation, $T_{C_{1}}=\left(x_{1}, \bar{x}_{4}, \bar{x}_{2}, x_{3}, x_{5}\right)$, and the canonical form, $f_{1}^{C}(X)=\left(x_{1}+x_{2}+x_{3}\right) \cdot\left(x_{4}+x_{5}\right)$, were given in example 5. For function $f_{2}$, the classes (after phase assignment) and the corresponding $1^{\text {st }}$-signatiures are: $C_{1}=\left\{x_{1}, x_{2}\right\}$ with $\left|f_{C_{1}}\right|=14$ and $C_{2}=\left\{x_{3}, x_{4}, x_{5}\right\}$ with $\left|f_{C_{2}}\right|=12$. Since $\left|f_{C_{2}}\right|<\left|f_{C_{1}}\right|$, the maximal transformation is $\left(C_{2}, C_{1}\right) \equiv\left(x_{3}, x_{4}, x_{5}, x_{1}, x_{2}\right)=T_{C_{2}}^{-1}$ and the canonical form of $f_{2}(X)$ is: $f_{2}^{C}(X)=f_{2}\left(T_{C_{2}}\right)=\left(x_{1}+x_{2}+x_{3}\right) \cdot\left(x_{4}+x_{5}\right)$ which is equal to the canonical form of $f_{1}: f_{1}^{C}(X)=f_{2}^{C}(X)$. Therefore, functions $f_{1}$ and $f_{2}$ are NP-equivalent.

In this part we consider the case where not all of the $1^{\text {st }}$-signatures are distinct, i.e., there exist two or more classes with the same $1^{\text {st }}$ signature. In this case the classes are placed in $k$ groups such that all classes inside a group have the same $1^{\text {st }}$-signature:

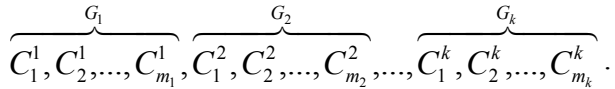

The superscript $j$ of a class $C_{i}^{j}$ indicates that it belongs to the group $G_{j}$. Note that $\left|f_{C_{1}^{j}}\right|=\left|f_{C_{2}^{j}}\right|=\ldots=\left|f_{C_{m_{j}^{j}}}\right|$ and groups are ordered based on the $1^{\text {st }}$-signatures, i.e., $\left|f_{C_{i}^{1}}\right|<\left|f_{C_{i}^{2}}\right|<\ldots<\left|f_{C_{i}^{k}}\right|$. This ordering of groups imposes a restriction on the $\mathrm{CP}$ transformation whereby all classes inside a group $G_{i}$ should precede classes of $G_{i+1}$ i.e., candidates for maximal transformation are transformations that respect the ordering of the groups.

Example 7. Consider the Boolean function of a six-input multiplexer: $f=\bar{x}_{5} \bar{x}_{6} x_{1}+\bar{x}_{5} x_{6} x_{2}+x_{5} \bar{x}_{6} x_{3}+x_{5} x_{6} x_{4}$. In this function there is no symmetry between variables i.e., each symmetry class contains only one variable. After doing phase assignment, ordering and grouping classes (in this case, variables), two groups $\overbrace{x_{i}, x_{6}}^{G_{1}:\left|f_{x_{i}}\right|=16} \overbrace{x_{1}, x_{2}, x_{i}, x_{4}}^{G_{2}:\left|f_{x_{i}}\right|=20}$

$G_{1}$ and $G_{2}$ are created: $\overbrace{x_{5}, x_{6}}, \overbrace{x_{1}, x_{2}, x_{3}, x_{4}}$ where for members in $G_{1}$ the phase is undecided since $\left|f_{x_{5}}\right|=\left|f_{x_{5}}\right|=\left|f_{x_{6}}\right|=\left|f_{x_{6}}\right|=16$ while for $G_{2}$ the phase is decided and the $1^{\text {st }}$-signatures are $\left|f_{x_{i}}\right|=20$. In the next step of the compute-CF algorithm, the $2^{\text {nd }}-$ signatures are used to order classes inside individual groups and then split the groups into smaller ones based on the outcome of ordering until each group contains only one class at which point a total ordering on classes has been obtained.

We refer to a group as unresolved if it contains more than one class or the phases of classes in that group are undecided. (Because all classes in the group have the same $1^{\text {st }}$-signature, the phase of all classes in that group is decided or none of the phases is decided.) More precisely, if $C_{1}^{j}$ and $C_{2}^{j}$ belong to the same group and $\quad\left|f_{C_{1}^{j}}\right|=\left|f_{\bar{C}^{\prime}}\right|, \quad$ then $\quad\left|f_{\bar{C}_{2}^{j}}\right|=|f|-\left|f_{C_{2}^{j}}\right|=$ $|f|-\left|f_{C_{1}^{j}}\right|=\left|f_{\bar{C}_{1}^{j}}\right|=\left|f_{C_{1}^{j}}\right|=\left|f_{C_{2}^{j}}\right|$.) Let $G_{u}=\left\{C_{1}^{u}, C_{2}^{u}, \ldots, C_{m_{u}}^{u}\right\}$ be the first unresolved group. Since all groups $G_{1}, G_{2}, \ldots, G_{u-1}$ are resolved, (i.e., they contain a single class with decided phase,) the ordering of classes up to $G_{u}$ is identified. (The case that $G_{1}$ is unresolved is discussed later.) Now the $2^{\text {nd }}$-signatures are used to specify the ordering inside the unresolved groups starting with $G_{u}$. Since $G_{1}$ is resolved, $G_{1}=\left\{C_{1}^{1}\right\}, 2^{\text {nd }}$-signatures with respect to $C_{1}^{1}$ and $C_{i}^{u}$ (i.e., $\left|f_{C_{1}^{1} C_{i}^{u}}\right|$ ) can be used for phase assignment (if needed) and ordering classes $C_{1}^{u}, C_{2}^{u}, \ldots, C_{m_{u}}^{u}$ (later on this step will be referred to as iteration 1.) If phase of a class $C_{i}^{u}$ is undecided, the $2^{\text {nd }}$-signatures $\left|f_{C_{1}^{1} C_{i}^{u}}\right|$ and $\left|f_{C_{1}^{1} \bar{C}_{i}^{u}}\right|$ are compared and if $\left|f_{C_{1}^{1} \bar{C}_{i}^{u}}\right|<\left|f_{C_{1}^{1} C_{i}^{u}}\right|$, then a positive phase is assigned to $C_{i}^{u}$; otherwise if $\left|f_{C_{1}^{1} C_{i}^{u}}\right|<\left|f_{C_{1}^{1} \bar{C}_{i}^{u}}\right|$, then a negative phase is assigned to $C_{i}^{u}$. In case of equality of the $2^{\text {nd }}$-signatures, the phase of $C_{i}^{u}$ remains undecided. Next, new values of $2^{\text {nd }}$-signatures $\left|f_{C_{1}^{1} C_{i}^{u}}\right|$ after phase assignment are used to order classes $C_{1}^{u}, C_{2}^{u}, \ldots, C_{m_{u}}^{u}$ and subsequently regroup these classes. Moore concretely, $G_{u}$ is split into smaller groups such that inside each group the $2^{\text {nd }}$-signatures, $\left|f_{C_{1}^{1} C_{i}^{u}}\right|$, are equal. The same procedure (phase assignment, ordering and regrouping based on $\left.\left|f_{C_{1}^{1} C_{i}^{v}}\right|\right)$ is applied to all other unresolved groups $G_{v}$. Finally, the indices of new groups and corresponding classes are properly updated. If after these steps, there still exist some unresolved groups $G_{w}$, a similar procedure (called iteration 2) is applied based on $2^{\text {nd }}$-signatures with respect to $C_{1}^{2}$ and $C_{i}^{w}$ (i.e., $\left|f_{C_{1}^{2} C_{i}^{w}}\right|$ ). If needed iterations $3,4, \ldots, u-2$ and $u$ 1 are applied. If at iteration $u$, there still exists some unresolved groups and $G_{u}$ itself is also unresolved, the procedure described below will be used. (This case includes the case where $G_{1}$ is unresolved.)

At this point, group $G_{u}=\left\{C_{1}^{u}, C_{2}^{u}, \ldots, C_{m_{u}}^{u}\right\}$ has been split into two groups such that one of them contains only one class and the other group contains the rest of $m_{u}-1$ classes: $\overbrace{C_{i}}^{G_{u}} \overbrace{C_{1}, \ldots, C_{i}, C_{i+1}}^{G_{u+1}}$

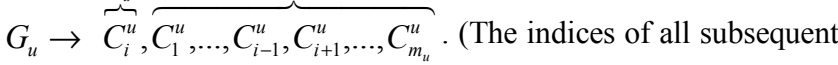
groups are shifted by one.) As can be seen, there are $m_{u}$ ways to split the group $G_{u}\left(m_{u}\right.$ ways to specify new $G_{u}$ corresponding to $i=1,2, \ldots, m_{u}$ in the above relation) and if the phase of $C_{i}^{u}$ is undecided, then there will be two ways to resolve the group, new $G_{u}$, (positive or negative phases.) Therefore there are $m_{u}$ (or $2 m_{u}$ ) ways to specify and resolve the new group, $G_{u}$. All these $m_{u}$ (or $2 m_{u}$ ) cases need to be tracked, since it is unknown which one(s) will result in a maximal transformation. For each case, the $2^{\text {nd }}-$ signatures are used to first order classes inside the unresolved 
groups among $G_{\mathrm{u}+1}, \ldots, G_{k}$ and then split them based on the outcome of ordering as follows.

Assume that $G_{u}$ is split into two following groups: $\overbrace{C_{i}}^{G_{u}} \overbrace{C_{1}, \ldots, C_{i-1}, C_{i+1}, G_{u+1}}^{G_{u}}$

$G_{u} \rightarrow \overbrace{C_{i}^{u}}^{u}, \overbrace{C_{1}^{u}, \ldots, C_{i-1}^{u}, C_{i+1}^{u}, \ldots, C_{m_{u}}^{u}}$, after shifting the indices of subsequent groups and making corresponding changes to superscripts of classes, new groups $G_{u}, G_{u+1}, \ldots, G_{k}$ (where $k$ represents the new number of groups) are represented as: $\overbrace{C_{1}^{u}}^{G_{u}} \overbrace{C_{1}^{u+1}, C_{2}^{u+1}, \ldots, C_{m_{u+1}}^{u+1}}^{G_{u+1}}, \ldots, \overbrace{C_{1}^{k}, C_{2}^{k}, \ldots, C_{m_{k}}^{k}}^{G_{k}}$

$u$ ) similar to the procedure explained before, for unresolved groups $G_{v}$, the $2^{\text {nd }}$-signatures $\left|f_{C_{1}^{1} C_{i}^{\nu}}\right|$ are used for phase assignment, ordering and regrouping. This process will continue for all $m_{u}$ (or $2 m_{u}$ ) cases, recursively (cf. the recursive-resolve algorithm), until all groups are resolved.

All resulting transformations $T_{1}, T_{2}, \ldots, T_{r}$ corresponding to different cases are stored. These transformations are superior with respect to relation ' $\prec$ ', considering only the $1^{\text {st }}$ and $2^{\text {nd }}$ signatures because of the way they have been constructed. The maximal transformation(s) is identified among these transformations. As a result of the way in which these transformations are obtained, they all have the same set of $1^{\text {st }}$ and $2^{\text {nd }}$ signatures. Some of these transformations are equivalent to each other because of special types of symmetry (excluding the type of symmetry discussed in section IV.) Examples of such special symmetries include hierarchical, group and rotational symmetries [4] i.e., for some $T_{i}$ and $T_{j}$, the equality $f\left(T_{i}^{-1}\right)=f\left(T_{j}^{-1}\right)$ may hold, in which case only one of $T_{i}$ and $T_{i}$ is kept and the other one is removed from the list $T_{1}, T_{2}, \ldots, T_{r}$. This process continues until there is no equivalency among the remaining transformations. Experimental results show that for nearly all Boolean functions only one transformation will remain in the list; i.e., all transformations $T_{1}, T_{2}, \ldots, T_{r}$ are equivalent: $f\left(T_{1}^{-1}\right)=f\left(T_{2}^{-1}\right)=\ldots=f\left(T_{r}^{-1}\right)$. In this case all transformations, $T_{1}, T_{2}, \ldots, T_{r}$ are canonical and the canonical form is $f^{c}(X)=f\left(T_{1}^{-1}\right)=\ldots=f\left(T_{r}^{-1}\right)$. In other words, in most cases, only the $1^{\text {st }}$ and $2^{\text {nd }}$ signatures can identify the maximal transformation(s).

Example 8. Continuing with the function of example 7,

$$
\begin{gathered}
f(X)=f\left(x_{1}, x_{2}, x_{3}, x_{4}, x_{5}, x_{6}\right)=\bar{x}_{5} \bar{x}_{6} x_{1}+\bar{x}_{5} x_{6} x_{2}+x_{5} \bar{x}_{6} x_{3}+x_{5} x_{6} x_{4} \mid: \overbrace{x_{x_{i}} \mid=16}^{G_{G_{2}}: \mid f_{x_{i} i} i=20}
\end{gathered}
$$

and variables are grouped as $\overbrace{x_{5}, x_{6}}, \overbrace{x_{1}, x_{2}, x_{3}, x_{4}}$. Both groups $G_{1}$ and $G_{2}$ are unresolved. Group $G_{1}$ can be split into two groups in two ways: $G_{1} \rightarrow \overbrace{x_{5}}^{G_{1}}, \overbrace{x_{6}}^{G_{2}}$ or $G_{1} \rightarrow \overbrace{x_{6}}^{G_{1}}, \overbrace{x_{5}}^{G_{2}}$. For each of these cases there are two ways to assign phase to $G_{1}$. Therefore, there are four

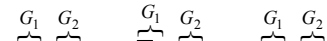

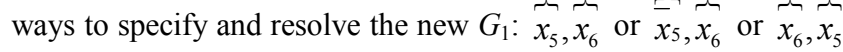
$\stackrel{G_{1}}{G_{2}}$

or $\stackrel{x}{x}_{6}, x_{5}$. The algorithm keeps track of all these cases. Let's zoom in one of the cases, e.g., $G_{1} \rightarrow \stackrel{G_{1}}{G_{x_{6}}}, \overbrace{x_{5}}^{G_{2}}$ results in the following new $\stackrel{G_{1}}{\sim} \stackrel{G_{2}}{G_{x_{6}}} \overbrace{x_{5}, x_{2}, x_{3}, x_{4}}^{G_{3}}$

grouping: $\overbrace{x_{6}}, \overbrace{x_{5}}, \overbrace{x_{1}, x_{2}, x_{3}, x_{4}}$. Now, $G_{2}$ and $G_{3}$ are unresolved. $G_{2}$ is unresolved because the phase of $x_{5}$ is undecided. First we try to resolve $G_{2}$ (decide a phase for $x_{5}$ ) using $2^{\text {nd }}$-signatures $\left|f_{\bar{x}_{6} x_{5}}\right|$ and $\left|f_{\bar{x}_{6} \bar{x}_{5}}\right|$. Since $\left|f_{\bar{x}_{6} x_{5}}\right|=\left|f_{\bar{x}_{6} \bar{x}_{5}}\right|=8$ the phase of $x_{5}$ can not be decided and the group $G_{2}$ remains unresolved. Next, we try to resolve $G_{3}$ using $2^{\text {nd }}$-signatures $\left|f_{\bar{x}_{6} x_{1}}\right|=12,\left|f_{\bar{x}_{6} x_{2}}\right|=8,\left|f_{\bar{x}_{6} x_{3}}\right|=12$ and $\left|f_{\bar{x}_{6} x_{4}}\right|=8$. (Recall that the phase of literals in $G_{3}$ is decided.) Since $\left|f_{\bar{x}_{6} x_{2}}\right|=\left|f_{\bar{x}_{6} x_{4}}\right|<\left|f_{\bar{x}_{6} x_{1}}\right|=\left|f_{\bar{x}_{6} x_{3}}\right|$, the group $G_{3}$ is split into two groups: $G_{3} \rightarrow \overbrace{x_{2}, x_{4}}^{G_{3}}, \overbrace{x_{1}, x_{3}}^{G_{4}}$ and the overall grouping will be

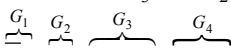
$\bar{x}_{6}, \overbrace{x_{5}}, \overbrace{x_{2}, x_{4}}, \overbrace{x_{1}, x_{3}}$. At this point, $G_{2}, G_{3}$ and $G_{4}$ are still undecided. There are two ways to resolve $G_{2}: \stackrel{G_{2}}{\overbrace{5}}$ or $\stackrel{G_{2}}{\overbrace{5}}$.

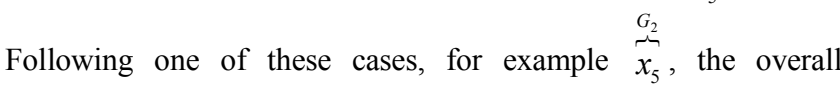
$\overbrace{x_{6}, x_{2}, x_{2}, x_{4}, x_{1}, x_{3}}^{G_{2}} \overbrace{G_{3}}^{G_{4}}$

grouping will be $\bar{x}_{6}, x_{5}, \overbrace{x_{2}, x_{4}}, \overbrace{x_{1}, x_{3}}$ where $G_{1}$ and $G_{2}$ are resolved and $G_{3}$ and $G_{4}$ are unresolved. Now we try to resolve $G_{3}$ using $2^{\text {nd }}-$ signatures $\left|f_{x_{5} x_{2}}\right|=8$ and $\left|f_{x_{6} x_{4}}\right|=12$. Since $\left|f_{x_{5} x_{2}}\right|<\left|f_{x_{6} x_{4}}\right|$, the group $G_{3}$ is split to $\stackrel{x_{2}}{x_{4}}$ and similarly for $G_{4}$ since

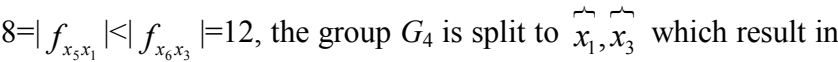

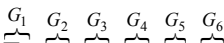
the following ordering of resolved groups: $\vec{x}_{6}, x_{5}, x_{2}, x_{4}, x_{1}, x_{3}$ which corresponds to the transformation $T_{1}=\left(\bar{x}_{6}, x_{5}, x_{2}, x_{4}, x_{1}, x_{3}\right)$. We only followed one case out of eight cases; (four ways to resolve $G_{1}$ multiplied by two ways to resolve $G_{2}$.)

Following all cases will result in overall eight transformation including $T_{1}$. Among other seven cases, one for example is $T_{2}=\left(x_{5}, x_{6}, x_{2}, x_{1}, x_{4}, x_{3}\right)$. However, it can be seen that:

$f\left(T_{1}^{-1}\right)=f\left(x_{5}, x_{3}, x_{6}, x_{4}, x_{2}, \bar{x}_{1}\right)=\bar{x}_{2} x_{1} x_{5}+\bar{x}_{2} \bar{x}_{1} x_{3}+x_{2} x_{1} x_{6}+x_{2} \bar{x}_{1} x_{4}$,

$f\left(T_{2}^{-1}\right)=f\left(x_{4}, x_{3}, x_{6}, x_{5}, x_{1}, \bar{x}_{2}\right)=\bar{x}_{1} x_{2} x_{4}+\bar{x}_{1} \bar{x}_{2} x_{3}+x_{1} x_{2} x_{6}+x_{1} \bar{x}_{2} x_{5}$.

Since $f\left(T_{1}^{-1}\right)=f\left(T_{2}^{-1}\right)=\bar{x}_{1} \bar{x}_{2} x_{3}+\bar{x}_{1} x_{2} x_{4}+x_{1} \bar{x}_{2} x_{5}+x_{1} x_{2} x_{6}$,

transformations $T_{1}$ and $T_{2}$ are equivalent. All eight transformations will result in the same function, which implies that any one of them, e.g., $T_{1}$, is a maximal transformation and the canonical form is $f^{C}(X)=f\left(T_{1}^{-1}\right)=\bar{x}_{1} \bar{x}_{2} x_{3}+\bar{x}_{1} x_{2} x_{4}+x_{1} \bar{x}_{2} x_{5}+x_{1} x_{2} x_{6}$.

If at this point, more than one transformation is left in the list; the $1^{\text {st }}$ and $2^{\text {nd }}$ signatures can no longer be utilized. For the remaining transformations $T_{1}, T_{2}, \ldots, T_{l}$, the partial signature vectors $P^{T_{1}}$, $P^{T_{2}}, \ldots, P^{T_{l}}$ are generated where partial means the $1^{\text {st }}$ and $2^{\text {nd }}$ signatures are excluded and only the $3^{\text {rd }}$ and higher order signatures are used. For example, if $T=\left(t_{1}, t_{2}, \ldots, t_{n}\right)$, then

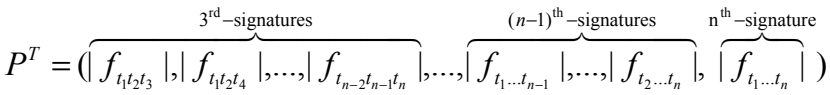
Since for all pairs $T_{i}$ and $T_{j}$ among $T_{1}, T_{2}, \ldots, T_{l}$, we have $f\left(T_{i}^{-1}\right) \neq f\left(T_{j}^{-1}\right)$, as a result of theorem 1 , all respective partial signature vectors $P^{T_{1}}, P^{T_{2}}, \ldots, P^{T_{l}}$ are different. Hence, a unique maximal transformation (with respect to partial signature vectors), $T_{C}$ (which will be canonical) can be identified among $T_{1}, T_{2}, \ldots, T_{l}$, In other words, the maximal transformation(s) has the maximal partial signature vector with respect to the lexicographical ordering ' $\prec$ ' discussed in section IV, among the transformations $T_{1}, T_{2}, \ldots, T_{l}$. For nearly all functions, there is no need to generate all signatures for identifying the maximal transformation(s) with respect to ' $\prec$ '. Instead, to compare two transformations $T_{i}$ and $T_{j}$, at the first step, only $3^{\text {rd }}$-signatures are generated and comparison is performed with respect to $3^{\text {rd }}$-signatures only. If the corresponding $3^{\text {rd }}$-signatures are equal, then $4^{\text {th }}$-signatures are generated and compared and this procedure continues until an inequality is encountered. As a result of theorem 1, it is guaranteed that at some point, an inequality will occur, because $f\left(T_{i}^{-1}\right) \neq f\left(T_{j}^{-1}\right)$. 
Different steps of the proposed techniques are summarized in the following pseudo-code descriptions of compute-CF and recursive-resolve algorithms.

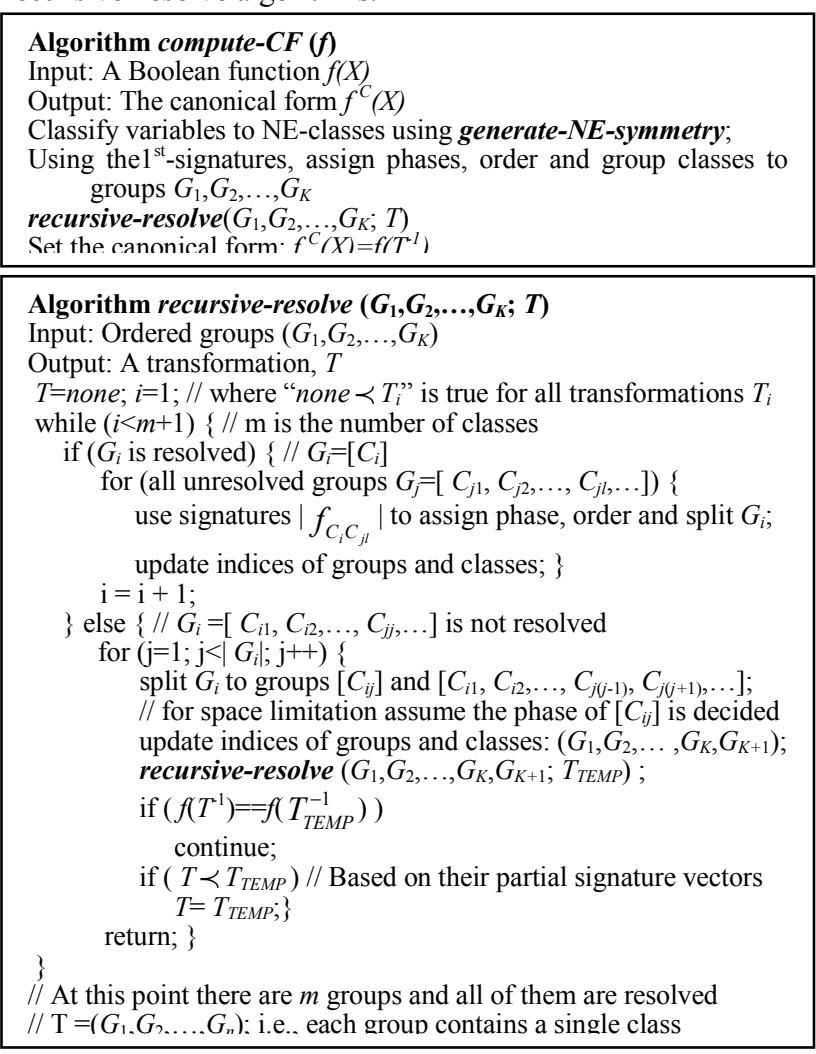

\section{Experimental Results}

The technique presented above has been implanted as part of the SIS logic synthesis environment. To reveal the effectiveness of the proposed technique, the proposed canonical form is computed for all cells in a cell library, containing a large number of complex cells with up to 20 inputs. To asses the efficiency of the method, a large number of randomly generated logic cells with different input counts were added to the library. Figure 1 shows the worstcase and average run-times required for computing the canonical form in terms of the number of inputs; i.e., the height of the $\mathrm{n}^{\text {th }}$ bars are the worst-case and average runtimes for all n-input cells.

The run-times in this Figure 1 (Y-axis) are in microseconds and include data for cells with more than five inputs. This is because run-times for cells with as few as five inputs are too small (less than $1 \mu-\mathrm{sec})$ to be discernible. As an example the worst-case 20input cell was a multiplexer with four select inputs for which the algorithm takes 240 microseconds to compute its canonical form.

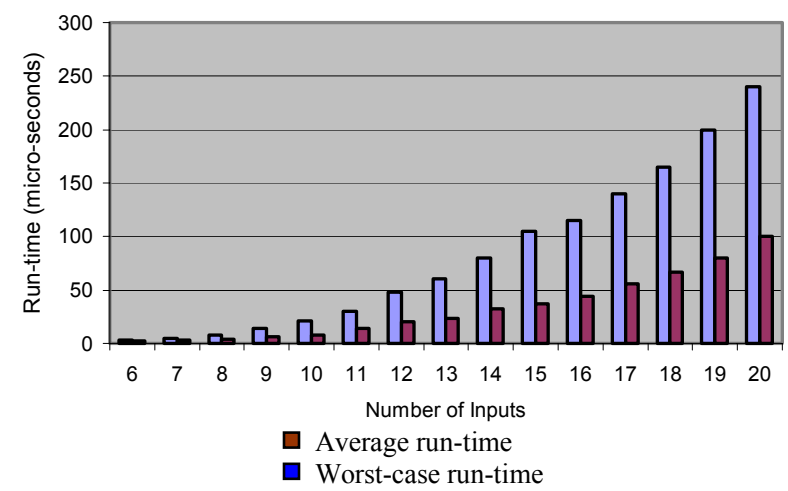

Figure 1. Worst-case and average runtime to compute canonical forms These results show a major improvement in run-time over previous approaches [9][10]. (Notice that reference [9] does not handle complementation of inputs and reference [10] entails enormous space complexity.) For nearly all of the cells in the library, the canonical forms were computed using only the $1^{\text {st }}$ and $2^{\text {nd }}$ signatures. Only one of the cells required the use of the $3^{\text {rd }}$ signatures and none of them required the use of higher order signatures. However, the algorithm given above is complete and able to handle functions that may require the use of higher order signatures for computing the canonical form.

\section{Conclusions}

A new efficient and compact canonical form was defined and an effective algorithm for computing the proposed canonical form was provided in this paper. The compactness and efficiency of the presented methods enables the approach to be applicable to a wide range of Boolean networks as apposed to previous approaches that either do not solve the problem generally or only handle functions with limited number of inputs. This paper addresses the general Boolean matching problem in which both permutation and complementation of inputs are considered. The proposed canonical form was based on using generalized signatures to obtain a CP transformation on inputs. Signatures were defined very effectively and first, most powerful signatures (that include more information about the function) are generated and used followed by less significant signatures, only if necessary. Experimental results demonstrate the efficiency of the proposed approach and it was observed, in nearly all cases $1^{\text {st }}$ and $2^{\text {nd }}$ signatures are enough to provide the canonical form and since handling $1^{\text {st }}$ and $2^{\text {nd }}$ signatures is performed efficiently by ordering variables, the proposed approach is associated with a very low computational complexity.

\section{Acknowledgment}

This work was sponsored in part by a grant from Magma Design Automation Inc.

\section{References}

[1] G. De Micheli, Synthesis and Optimization of Digital Circuits, McGraw-Hill, 1994.

[2] L. Benini and G. De Micheli, "A survey of Boolean matching techniques for library binding," ACM Trans. Design Automation of Electronic Systems, vol. 2, no. 3, pp. 193-226, July 1997.

[3] M. A. Harrison, Introduction to Switching and Automata Theory, McGraw-Hill, 1965.

[4] J. Mohnke, P. Molitor, and S. Malik, "Limits of using signatures for permutation independent Boolean comparison," Proc. of ASP Design Automation Conf., pp. 459-464, 1995.

[5] J. R. Burch and D. E. Long, "Efficient Boolean function matching," in Proc. Int. Conf. on Computer-Aided Design, pp. 408-411, Nov. 1992.

[6] Q. Wu, C. Y. R. Chen, and J. M. Acken, "Efficient Boolean matching algorithm for cell libraries," Proc. IEEE Int. Conf. on Computer Design, pp. 36-39, Oct. 1994.

[7] U. Hinsberger and R. Kolla, "Boolean matching for large libraries," Proc. of Design Automation Conf., pp. 206-211, June 1998.

[8] D. Debnath and T. Sasao, "Fast Boolean matching under permutation using representative," Proc. ASP Design Automation Conf., pp. 359362, Jan. 1999

[9] J. Ciric and C. Sechen, "Efficient canonical form for Boolean matching of complex functions in large libraries," IEEE Trans. Computer-Aided Design of Integrated Circuits and Systems, vol. 22, no. 5, pp. 535-544, May 2003.

[10]D. Debnath and T. Sasao, "Efficient computation of canonical form for Boolean matching in large libraries," Proc. ASP Design Automation Conf., pp. 591-596, Jan. 2004.

[11] C. R. Edwards and S. L. Hurst, "A digital synthesis procedure under function symmetries and mapping methods", IEEE Trans. Comp., Vol. C-27, No. 11, pp. 985-997, NOV. 1978. 